\title{
A Narrative Review
}

\section{Immunological and Neurological Manifestations of COVID-19: An Intimate Relationship}

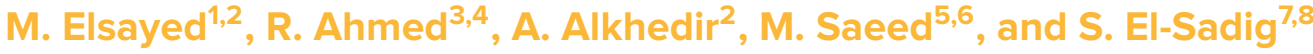 \\ ${ }^{1}$ Department of Neurology, Omdurman Islamic University Khartoum, Sudan \\ ${ }^{2}$ Sharjah University Hospital, Sharjah, UAE \\ ${ }^{3}$ Ahfad University for Women Khartoum Sudan \\ ${ }^{4}$ University of Sharjah, Sharjah, UAE \\ ${ }^{5}$ Department of Neurology, Altagana University, Khartoum, Sudan \\ ${ }^{6}$ National Center for Neurological Sciences, Khartoum, Sudan \\ ${ }^{7}$ Department of Neurology, Khartoum University, Khartoum, Sudan \\ ${ }^{8}$ Soba University Hospital, Khartoum, Sudan
}

Corresponding Author:

M. Elsayed

email: muaz.elsayed@uhs.ae

Received 12 May 2020

Accepted 24 July 2020

Published 29 July 2020

Production and Hosting by

Knowledge E

(c) M. Elsayed et al. This

article is distributed under the

terms of the Creative

Commons Attribution

which permits

unrestricted use and

redistribution provided that

the original author and

source are credited.

Editor-in-Chief:

Prof. Mohammad A. M. Ibnouf

\section{Abstract}

Background: COVID-19 has superseded all medical scientific challenges in 2020. It presented with a range of respiratory involvement from the mild upper respiratory tract to severe pneumonia with ARDS. A percentage of these patients manifested variable neurological presentations expanding the challenges of patients' assessment, care, and management.

Objective: To discuss the neurological manifestations and the possible immunopathology of COVID-19.

Methods: A literature search was performed in the PubMed database for the relevant articles published in English language between April 9 ${ }^{\text {th }}, 2020$ and June 1, 2020. Few papers were extracted from Google Scholar and pre-print material as well. The keywords used to perform search included "COVID-19," "SARS Cov2," "neurological manifestations," and "immunology and pathology of COVID-19". However, animal studies were excluded in the neurological manifestations, and the final number of literature search outcome was 27 articles.

Results: The immunopathology involves angiotensin-converting enzyme (ACE) receptor 2 and spike protein S1. COVID-19 has a tremendous affinity to the ACE2 receptor. The status of secondary hemophagocytic lymphohistiocytosis (sHLH) and the cytokine storm lead to different organ damage and the corresponding clinical manifestations. The documented neurological manifestations mainly include acute ischemic stroke, hemorrhagic stroke, temporal lobe, thalamic encephalitis, and one case of Guillain-Barre Syndrome, in addition to agitation, headache, and seizures.

Conclusion: Although the intimate relationship between the neurons and the immune activation is the basic concept of COVID-19 immunopathology, the definite routes of entry to the neuronal cells are yet to be disclosed. Acute stroke, myositis, headache, and meningoencephalitis are the most common types of complications of COVID-19 so far. 


\section{Introduction}

COVID-19, which caused a global lockdown in 2020, exerts a major health and economic challenge. Starting at the molecular level, the genome of Corona Virus Disease 2019 (COVID-19) has many similarities with the severe acute respiratory syndrome coronavirus (SARS-CoV) that caused the epidemic of 2003. Hence, the WHO initially named it as SARS-CoV-2, which was later changed to COVID-19. Both viruses share the same receptors to enter the cells that are the angiotensin-converting enzyme 2 receptor [1].

The major organs affected by COVID-19 are the lungs with rapidly fatal pneumonia, occasionally requiring ventilatory support. The brain expresses a metallopeptidase, ACE2 receptors in the glial cells and neurons. This can make the neurons a possible goal for COVID-19 virus [2, 3]. The objective of this review was to discuss the neurological manifestations and the possible related immunopathology of COVID-19.

\section{Methodology}

For this narrative review, articles published in English language between April $9^{\text {th }}, 2020$ and June 1, 2020 were searched in PubMed database. A review of the bibliographies from Google Scholar was performed to identify the supplementary papers that were not originally found in the initial search. The terms "COVID-19" and "SARS Cov2" were used and searched for the last five years. Only human studies for all ages and countries were included in this study. Next, a search for immunology and pathology of COVID19 was done using the same inclusion criteria. Further, a cross-search using the term "COVID-19" and "neurological manifestations" revealed some retrospective descriptive studies from Wuhan, China and a few case reports. Two review articles were included, in addition to few pre-print articles that met our search criteria. Moreover, the literature with negative serology of COVID-19 and literature in other languages were excluded. Animal studies were also excluded in the neurological manifestations. The final number of literature search outcome was 27 articles.

\section{Results}

\subsection{Immunopathology}

The process involves activation of both innate and adaptive immunity. The ACE2 receptor affinity for SARS-Cov2 is mediated by the spike protein S1 which facilitates the virus attachment to the cell wall. This receptor is expressed in the human airway epithelia, lung parenchyma, vascular endothelial cells, kidneys, cardiac, testicular, and small intestine. Another coronavirus, the Middle East respiratory syndrome (MERS-CoV) enters human cells through Di-peptidyl peptidase 4 (DPP4) in the lower respiratory tract and other tissues. COVID-19 has an average of 15-fold higher affinity for the spike protein S1 than in SARS-Cov [1]. Hence, COVID-19 virus makes use of the ACE 2 receptors to facilitate its cell entry to various target organs. The aforementioned proteins are not the only mechanisms of virus cellular entry as some tissues failed to demonstrate the 
presence of ACE2 receptors but still got the virus documented such as some endothelial and intestinal cells [2].

The immune system response in infectious conditions may differ from one human to another depending on various genetic, racial, or environmental factors. However, some will develop a secondary hemophagocytic lymphohistiocytosis (sHLH). During this hyperinflammatory immune syndrome, there is a fatal and fulminant hypercytokinemia. This is usually triggered by viruses in adults [4] and complicates around $4 \%$ of sepsis cases [5]. Moreover, major cytokines may be involved in this process like IL-6, IL-1beta, IL-2, IL-8, IL-17, G-CSF, GM/CSF, IP-10, MCP1, MIP1 alpha (CCL3), and TNF [6].

The mode of entry of the virus to the central nervous system (CNS) harbors many theories such as the direct invasion, hematogenous/lymphatic spread, peripheral nerve invasion, and trans-synaptic transmission [2]. Moreover, the ARDS patients are critically ill patients and during their illness, they were noticed to have confounding metabolic and toxic factors adding or creating an encephalopathic state. Other coronaviruses may cause generalized motor seizures or subclinical epileptic activity in a less common fashion $[7,8]$. The inflammatory process may induce a thrombotic cascade leading to a stroke. This is mediated by multiple factors which may include increased D-Dimers, accelerated atherosclerosis, and dehydration [9]. The elevated D-Dimers are recognized as a characteristic laboratory finding in COVID-19 infection. The C-reactive protein is also elevated. Around 5\% of COVID-19 patients develop ARDS, septic shock, and/or multiple organ failure $[6,10]$. They show a status of exhausted lymphocytes and lymphopenia with a reduced number of CD4 T cells, CD8 T cells, B cells, and natural killer (NK) cells. Also, the number of basophils, eosinophils, and monocytes are reduced [11]. Both innate and adaptive immunities are activated with uncontrolled responses contributing to tissue damage [12]. The condition exerts an increased IgG response and higher titers of all antibodies; this status of antibody immune incontinence is associated with the worst outcome [13]. Hence, this suggests an antibody-dependent enhancement (ADE) of COVID-19 infection.

Besides, trans-nasal inoculation of SARS-Cov or MERS-Cov caused brain involvement in transgenic mice mainly in the thalamus and brainstem. This caused high mortality in infected mice without demonstration of lung involvement. In contrast to avian influenza virus inoculation intranasally, SARS-Cov2 caused both brainstem and pneumonia.

The affected nuclei included the nucleus ambiguous and nucleus of the solitary tract. The human body breathing reflex includes the action of the mechanoreceptors and chemoreceptors in the lungs which sends their afferent impulses to the solitary tract nucleus. The efferent fibers emerge from the solitary tract nucleus and nucleus ambiguous to the airway smooth muscles, glands, and vascular bed [2]. This reflex is interrupted during the viral infection by coronaviruses due to nuclei infection which echoes the neuroinvasive tendency of the coronaviruses.

While in mice, the SARS-CoV managed to cause neuron death in the brain cells through entry by the nose, cribriform plate, and olfactory bulb, in humans, the exact route of entry to the neural cells by the SARS-Cov and MERS-Cov is not yet confirmed. It is unlikely that it is through hematogenous or lymphatics because no virus was detected in the non-neuronal tissues. However, there is some evidence that coronaviruses may 
enter through the peripheral nerves and travel to the CNS through synapses. Due to similarities in the genomic structure, receptor vulnerability, respiratory involvement, the SARS-Cov model can be used to suggest possible routes of entry to the neuronal cells in COVID-19.

\subsection{Neurological manifestations}

The neurological manifestations in the brain for COVID-19 patients are still emerging as the case fatality rises in Europe and USA. Coronaviruses can cause multiple systematic involvements in various animals including humans. Along with the multiple factors which add to the CNS involvement stand the elevated D-Dimers. The risk of a cerebrovascular event is high during these changes, especially in the microcirculation which will be liable to thrombosis or bleeding. The poor outcome in COVID-19 infection may be related to age $<40$ or $>60$ years, high pyrexia, CRP, neutrophil counts, elevated liver enzymes, high serum creatinine, and low platelets [14].

A pioneer pre-print work in Wuhan gave an overall view of neurology and COVID-19 as it described the neurological involvement in 214 COVID-19 patients through a review of the electronic medical records. These were categorized into CNS, peripheral nervous system (PNS), and muscle involvement [15]. The most common symptoms included dizziness (16.8\%) and headache (13.1\%). The diagnoses of CNS involvement depend on the $\mathrm{CT}$ findings, while muscle injury was determined by myalgia and Creatinine Kinase $\mathrm{CK}$ level $>200 \mathrm{U} / \mathrm{L}$. The latter may be attributed to the skeletal muscles vasculitic process in COVID-19 as in SARS [16]. While the PNS involvement included the symptomatology of hypogeusia (poor/loss of taste) without congestion and early hyposmia (reduced sense of smell) [17, 18], in the same retrospective study, $24.8 \%$ had CNS involvement, $8.9 \%$ had PNS, and 10.7\% had skeletal muscle involvement [15]

Of all the patients, neurological manifestations were reported in $36.4 \%$ and were more common in patients with severe infection, multiple co-morbidities (47\%), mainly hypertension $(\mathrm{HT})$ and atypical symptoms ( $P$-value $<0.05)$. Almost $45 \%$ of the patients with severe infections developed neurological symptoms.

Specific disorders in these patients with severe infection included acute cerebrovascular disease in $5.7 \%$ ( $80 \%$ of whom had an ischemic stroke), impaired consciousness in $14.8 \%$, and muscle injury in $19.3 \%$. All had significant $P$-values [15]. It is worth noticing that the ischemic brain injury seems to follow the usual expected vascular pathology in stroke patients being more ischemic than hemorrhagic. We expect this retrospective study to be the cornerstone in the clinical context of COVID-19 neurology. This is because it occurred in the early days of the virulent virus and when potential drugs were unknown compared to a few months later. Therefore, immune system of those patients' was left to defend the virus only with the supportive measures.

The initial stroke percentage reported from Wuhan was $5.7 \%$ in which the majority was ischemic in nature in severe cases. One percent of non-severe COVID-19 patients developed a stroke. Older people with co-morbidities were more prone to develop a cerebrovascular event than younger patients with COVID-19 infection [9]. One case report of a middle-aged female presenting with the classical symptoms and positive 
COVID-19 serology had associated confusion. The CT Brain showed bilateral hypoattenuation signals on the thalami. The MRI showed "evidence of hemorrhagic rim enhancing lesions within the bilateral thalami, medial temporal lobes, and sub insular regions" [19].

The ALBACOVID registry which was recently published included a larger group of patients (841) in Spain and stroke occurred in 1.7\% of patients. These were severe cases [20].

In one report of large vessel acute stroke in five young (below 50 years of age) COVID19 patients, the National Institute of Health and Stroke Scale (NIHSS) mean score was 17, indicating severe disability. Four patients had reduced consciousness level and one had hemiplegia. Only one patient was discharged home and the others were in ICU or rehabilitation units [21]. Hence, both large and small vessels are liable to vascular insults (vasculitis) reflecting their tendency to be caught in the severe inflammatory storm caused by COVID-19 infection. The radiological changes have proved involvement of different anatomical/vascular brain structures. This may suggest direct virus invasion of brain tissues as reported in MERS-Cov/ SARS-Cov.

As the COVID-19 puzzle is unfolding slowly, emerging neurological recommendations are coming through on the same speed. In the acute ischemic stroke setting, after the activation of the stroke code in case of the available stroke team, or if not, one doctor or resident with full PPE (personal protective equipment) should be exposed to the suspected case till the patient returns from radiology. This will reduce the risk during donning and doffing of PPE. If thrombolysis was indicated, the same doctor will remain inside the room while the patient is in radiology and during the first hour of Tissue Plasminogen Activator (TPA) infusion. Monitoring the patient requires filling of the q15 minute neuro-check; this can be performed by the doctor/resident/nurse inside the room. The clinical (including NIHSS) and imaging results should be discussed over the phone with the neurologist. In high-risk patients presenting with impaired GCS or large hemispheric infarctions, remote clinical assessment through video techniques (iPAD/Phone) should be performed. However, in high- or low-risk groups, concerns of bedside assessment can be revealed by the bedside neurologist assessment with PPE [22]. It is important to add here that there are no concerns about the inter-rater reliability in NIHSS assessment between different persons as it was appraised previously [23].

In the post-thrombectomy patients via catheter intervention, remote checks of the groin complications may be performed according to the recommendations and to avoid the risk of COVID-19 infection [24]. Imaging repetition is not generally recommended [22].

The other workup package includes all other relevant tests like Trans Esophageal Echo (TEE), Carotid Doppler, and Brain MRI should be delayed unless there is an endocarditis. It is also worth knowing that in general for acute stroke patients, the MRI is unlikely to exert major management changes [25]. A recent French study reported a bilateral frontotemporal hypoperfusion in $18 \%$, asymptomatic lacunar strokes in $3 \%$, and an established stroke in 1.7\% of COVID-19 patients [26].

In cases of intracranial hemorrhage, the same initial steps apply here. The follow-up CT, in general, may be done $3-6 \mathrm{hr}$ later as up to $40 \%$ of stroke patients may develop 
a clinically significant expansion of hematoma [27]. The prognosis will be worsened in case of COVID-19 positive patients due to the involvement of other organs.

Severe neurological involvement in the brain has been documented before in MERS CoV [28]. Hence, in our expectations, few COVID-19 cases may develop subarachnoid hemorrhage $(\mathrm{SAH})$ due to accelerated atherosclerosis and the effect of the inflammation on the stability of intracranial aneurysms [29]. The management of these patients should consider, in addition to the aforementioned points, the maintenance of euvolemia to prevent overload in ARDS, early intubation for large SAH, shorten ICU stay/early external ventricular drainage (EVD) clamp (avoid vasospasm), and the use of prophylaxis against DVT as COVID-19 patients have higher coagulability state [22].

The conclusion for acute stroke patients in the setting of COVID-19 infection is that it is more encountered in severe COVID-19 infection and they are mainly ischemic in nature with high disability. Specific management largely depends on the consultation of the stroke team. Then, the benefit-risk ratio should be assessed in the scope of infection control. The protocol recommends using teleneurology to minimize exposure, decide on the frequency of assessments, and relevant radiology for stroke in these highly infectious patients [22].

The severe inflammatory response causes ARDS in half of the patients. Hence, it can result in hemorrhagic encephalitis affecting various areas in the brain [30]. In a newer study observing neurological manifestations of SARS2 in an ICU in Strasbourg France, agitation was reported in 69\% of patients enrolled. Before the ICU admission, $14 \%$ had neurological manifestations, while $67 \%$ were observed to have it after cessation of neuromuscular block and sedation. This is not easy to be solely due to the virus effect on the brain as many metabolic, pharmacological, and inflammatory factors are involved. A high percentage (67\%) developed diffuse pyramidal signs with hyperreflexia and clonus. At the time of discharge, $34 \%$ had executive disturbances, inattention, disorientation, and disorganized movements. The encephalopathic features necessitated MRI imaging which showed leptomeningeal enhancement in $13 \%$. The leptomeningeal signs and the frontotemporal signs may support the neuroinvasive character of the virus. However, the final PCR results did not support this. The Electroencephalogram (EEG) showed nonspecific changes in $14 \%$. Cerebrospinal fluid (CSF) was acellular in $12 \%$, while matched oligoclonal bands (OCB) were reported in 3.4\%. Only one patient had high protein and IgG levels in the CSF. In $12 \%$ of the patients, the CSF PCR tested negative for COVID19 [26]. It seems that the variation of CSF immune and cytology findings reflects the variable immune mechanisms by which the virus may affect the neural tissues.

The first case to be reported as associated with encephalitis was described in a 24-year old man. His symptoms started with headache, fever, and malaise. On the second day, the patient was reviewed and treated as influenza by Laninamivir plus antipyretics. However, on day five, the patient deteriorated with a sore throat and worsening headache. The blood tests and CXR were reassuring. On the ninth day, he was found collapsed with surrounding vomitus. In the ER he presented with low GCS and developed a generalized motor seizure which recurred later multiple times. The patient had neck stiffness indicating meningio-encephalitis. There was evidence of neutrophil leukocytosis with leukopenia and high CRP. His nasopharyngeal swab 
for COVID-19 was negative but the CT chest was suggestive. Lumber puncture (LP) showed a clear CSF with high opening pressure $>320 \mathrm{mmH} 2 \mathrm{O}$, with cells of $12 / \mu \mathrm{L}(10$ mononuclear and 2 polymorphonuclear). No antibodies against Herpes were detected, however, the RNA of COVID-19 was detected in the CSF. On day one, the brain MRI diffusion-weighted images showed hyperintensities along with the inferior horn of the lateral ventricle on the right side. The Fluid Attenuation Inversion Recovery (FLAIR), on the other hand, showed hyperintense signals on the medial temporal lobe and hippocampus with slight hippocampal atrophy. This also documents the meningeal component besides the encephalitis process. This represents the first documented case of COVID-19 encephalitis with negative nasopharyngeal swab testing by PCR [31]. It seems that there is a predilection for the temporal lobes as herpes simplex virus but this is too early to be generalized. A single case of encephalitis was also reported in the ALBACOVID registry.

Currently, there are no clear guidelines for the treatment of the encephalopathic state in COVID-19 patients. The general measures apply and further steps depend on the outcome of relevant neuro-medical tests and the decision of the neurologist.

The pioneering study of 214 patients in Wuhan reported headache to be a symptom in 13.1\%. This is quite similar to $14.1 \%$ of the ALBACOVID registry. On the other hand, a study enrolled 62 patients and although headache as a symptom was higher (34\%), the later study targeted patients with human-to-human transmission only [32]. In a retrospective study in Wuhan which enrolled 41 positive patients for COVID-19, 8\% had a headache [6]. However, in the largest study with 262 patients, headache was reported in $6.2 \%$, and it was the lowest among all studies [33]. In a smaller group of 30 medical workers, headache was reported in 53.3\% [34]. The headache symptom was also reported in the first case of encephalitis with COVID-19. The variation reported in headache reflects that headache is a common presenting symptom of COVID-19 infection and the average percentage may be around $25 \%$. The management of chronic headache types with acute exacerbation in this setting is by using a combination of IV paracetamol, triptans, and different antiemetics. The use of opioids should be avoided so as not to degrade the respiratory effort. The symptomatic treatment by NSAIDs is not carrying a sufficient risk to worsen the infection depending on the available literature evidence [35]. Further evaluation of headache is needed if there are changes in the neurological examination or GCS or if it is a thunderclap headache. In these situations, the Boston Medical Center recommends proceeding for brain CT and LP if SAH is suspected. We think that the primary cause of headache in COVID-19 infection is not easy to separate as being a manifestation of the systemic immune changes or the direct involvement of the brain as proved in few cases either by imaging or CSF analysis. The accumulation of more headache-related literature in the setting of COVID-19 patients may reveal the final result.

Muscles constitute a large bulk of human body and are expected to be involved in the severe inflammatory process of COVID-19. While most of the literature has reported myalgia as a common feature of the infection that may occur in many viruses, where in one study, it was found in $44 \%$ of the patients [6], a lower percentage of $17.2 \%$ was reported from the ALBACOVID registry [20]. Another case report on an elderly female 
with multiple co-morbidities reported myalgia starting early in her deadly COVID-19 infection. Myalgia was also reported in middle-aged female air workers as a common symptom. In most of them, the Creatinine Kinase (CK) levels were $>200$ IU plus high inflammatory markers. This suggests a degree of muscle damage (myositis). It may not be severe enough to cause primary muscle weakness but the common occurrence and the persistence and associated elevation of muscle enzyme makes it a genuine immune characteristic of the virus and extends its target cells from neurons to muscle fibers.

The real epidemiological statistics of seizures in COVID-19 are still to be determined. However, in other coronaviruses' infections, seizures were estimated to range between $6 \%$ and $50 \%$ [7]. The recent report which reviewed 841 patients in Spain reported rarity of seizures in COVID positive patients as the percentage was only $0.7 \%$ [20]. It is expected that some will manifest clinical motor or other subclinical seizures. The underlying pathophysiology will range between the direct invasion in areas like temporal lobes, the disturbed hemostasis, and metabolic state. The former structure is well-known for its association with focal to bilateral sensory-motor seizures, and the stressful immune activation associated with COVID-19 may easily affect the electrical stabilization of this area. Moreover, the relevant imaging showed involvement of temporal lobes in some cases either on one or both sides. The Boston Medical Center recommendations in the management of the acute motor seizures in COVID- 19 follows the standard protocol with caution in cases with liver or kidney injuries. In liver disturbances, Brivaracetam, lacosamide, levetiracetam, or zonisamide may be preferred. The EEG recording should be discussed with the technician and the neurologist first to meet the fundamental goal of risk reduction. Patients with impaired consciousness may need continuous EEG monitoring to detect subclinical seizures, and this needs to be tailored according to the risk-benefit ratio [22].

The loss of taste and smell proved common neurological presentation in even mild disease. The ALBACOVID reported anosmia in 4.9\% and dysgeusia in 6.2\% [20].

For patients with suspected Guillain-Barre Syndrome (GBS) with COVID-19 infection, the initial assessment follows the standard neurological assessment with special focus on the signs of respiratory failure and pulmonary function testing. The point of care is preferred to be the ICU especially if we considered the risk of dysautonomia. The relevant investigations like Nerve Conduction Studies (NCS) and LP may be delayed if the clinical diagnosis is obvious as these tests are equivocal in up to $50 \%$ of cases in the first week. Moreover, the high risk in contracting the infection from these patients may have affected the detailed electrophysiological classification of myoneuropathy in them [36]. The standard GBS therapeutic measures apply in COVID-19 situation [22]. The options include the intravenous immune globulins infusions or the plasma exchange.

Till April 12, 2020, only one case assumed to have an acute GBS associated with COVID-19 infection was reported. The case scenario mentioned that four days after returning from the index city of Wuhan, a 61-year-old lady developed remarkable fatigue and acute lower limbs weakness. This was not accompanied by the cardinal symptoms of COVID-19 infection or respiratory failure. Her signs included power 4/5 muscle weakness, absent reflexes, and abnormal light touch and pin prick sensations. The NCS confirmed a form of demyelinating neuropathy while the blood counts showed 
lymphopenia and thrombocytopenia. After receiving the intravenous immune-globulins (IVIG), on day 8 she started to develop fever, dry cough, typical radiological features on CT scan as ground-glass appearance, and tested positive for COVID-19. In the third week, her blood counts normalized, and by the fourth week, she recovered completely including the tendon reflexes in the four limbs. The authors discussed the casual association in this case between GBS and COVID-19 in detail as the parainfectious rather than post-infectious neuropathy in this setting. However, they did not explain the sensory signs which are recognized in a small percentage of GBS subtype and the NCS which is expected to be normal in the first week in a significant percentage of patients. Hence, we believe that this may represent other para-infectious sensory-motor neuropathy which seems to be mild with good recovery. Since GBS is an autoimmune disease and molecular mimicry is the main immunopathological mechanism in its etiology, we find it difficult to confirm this case given the lack of CSF and autoantibodies result. The recruitment of other similar cases may reveal the ambiguity of the association [37]. However, in June, one case of GBS was described in the ALBACOVID registry [20]. The same study reported myopathy in 3.1\% and dysautonomia in 2.5\%. In conclusion to GBS and Covid-19 the association seems to be a rare one.

Despite that the prevention may lay out of the scope of this narrative review, COVID-19 exerted strict health and economical prevention methods. Neurology is not an exception in this worldwide trend.

The prevention protocol is essential for patients with known PNS disorders as they can contract the infection from the community or their caregivers. The adherence to the international/national guidelines of movement restriction, use of PPE, and restriction of hospital follow-up are of paramount importance. In the case of seropositive test, any pre-existing neurological disease will exert its negative effect on the outcome and the intensive care measures. This can be hypothetically generalized for other similar conditions especially hereditary and congenital muscle diseases. A target group here may include patients with Myasthenia Gravis, motor neuron disease, neurologic respiratory involvement, and kyphoscoliosis, all of which tend to have a poor respiratory reserve escalating morbidity and mortality profile. Patients with $<60 \%$ predicted FVC (forced vital capacity) are unlikely to wean from ventilation and patients with $<40 \%$ FVC are likely to be on NIPPV (noninvasive positive-pressure ventilation) already [38]. The septic status and prolonged stay in the ICU will increase the likelihood of critical illness neuromyopathy which adds to the negative outcome and functional disabilities [39]. Neurological complications may emerge due to non-virus causes like hypoxia, secondary hypercoagulable state, disseminated intravascular coagulopathy, and drug toxicities.

Another emerging puzzle is the use of immunosuppressive during COVID-19 infections in patients with multiple sclerosis (MS), autoimmune neuropathy, and autoimmune encephalitis that are susceptible to immune-related complications. These diseases represent the peak of neuronal immune activation with many questions pending solution and made worse by COVID mystery. Medications like high-dose steroids, Natalizumab (Tysabri), other monoclonal antibodies are yet to be investigated for their role in adding to the immune disturbance/mortality in concomitant COVID-19 infection. The association 
of British Neurologist released their recommendations about the safe use of Natalizumab, Dimethyl Fumarate (Tecfidera), Interferon beta 1a \& 1b, Teriflunomide, and Glatiramer acetate. While the Fingolimod (Gilenya) carried a moderate risk, a significant risk was suggested with the Alemtuzumab, Cladribine (Mavenclad), Ocrelizumab, Rituximab, Siponimod, and Ofatumumab. This may not match the recommendations for individual drugs in other publications [40]. A conservative recommendation indorses cautious use of highly efficient medications. This may be paralleled by rebound activation of the disease, making the balance difficult [41].

Many strategies were recommended to treat different categories of neurological patients during the pandemic of COVID-19. This included the hereditary ataxia patients especially during the prevention and control period of COVID-19 fatal pneumonia. Elderly with late-stage dementia, and young syndromic patients are also at high risk of complications. Other minor neurological symptoms encountered by the authors and related to the COVID-19 situation rather than the virus itself included self-limiting sensory ulnar neuropathy due to remote overworking from home, postauricular pain due to N95 straps, and mask-induced headaches.

All the navigated neurological manifestations during this infection may reflect the diversity of the symptomatology or the complications of the pre-existing diseases in such group of patients which constitutes a high risk for COVID-19.

\section{Conclusion}

The extreme immunological storm caused by the virus results in a secondary hemophagocytic lymphohistiocytosis (sHLH) ending with serious pathological damage to the lungs, brain, muscles, and kidneys. The virus has a huge affinity to the ACE 2 receptor placing the brain as a target organ. Headache is a common symptom of COVID-19 infections and affects around one-quarter of cases while myalgia affects almost half of them. Stroke in the setting of COVID-19 infection can affect both large vessels in $<50$ years and small vessels in older patients with severe infection. Intracerebral hemorrhage is less common. The primary pathology for stroke seems to be vasculitic as well as for the muscles. The encephalopathic features may be multifactorial but at least in part are strongly related to the neuroinvasion by the virus as previously documented in MERS-CoV and SARS-CoV. This is supported by abnormal CSF findings in some of these patients and positive PCR for COVID-19. Seizures may be caused by affection of the temporal lobes as shown in some cases. PNS involvement is uncommon in COVID-19 infection, while the loss of smell and taste are common symptoms even in patients with mild infection. The later symptoms may support the route of entry of the virus being near to the cranial neural structures. The final epidemiological pattern for the neurological manifestations of COVID-19 is yet to be revealed after the pandemic fades away. 


\section{Disclaimer}

The views mentioned in the conclusion represent the professional authors' opinions and not their corresponding institutions.

\section{References}

[1] Baig AM, Khaleeq A, Ali U, Syeda H. Evidence of the COVID-19 Virus Targeting the CNS: Tissue Distribution, Host-Virus Interaction, and Proposed Neurotropic Mechanisms. ACS chemical neuroscience. 2020;11(7):995-8.

[2] Li YC, Bai WZ, Hashikawa T. The neuroinvasive potential of SARS-CoV2 may play a role in the respiratory failure of COVID-19 patients. J Med Virol. 2020.

[3] Li W, Moore MJ, Vasilieva N, Sui J, Wong SK, Berne MA, et al. Angiotensin-converting enzyme 2 is a functional receptor for the SARS coronavirus. Nature. 2003;426(6965):450-4.

[4] Ramos-Casals M, Brito-Zeron P, Lopez-Guillermo A, Khamashta MA, Bosch X. Adult haemophagocytic syndrome. Lancet. 2014;383(9927):1503-16.

[5] Karakike E, Giamarellos-Bourboulis EJ. Macrophage Activation-Like Syndrome: A Distinct Entity Leading to Early Death in Sepsis. Frontiers in immunology. 2019;10:55.

[6] Huang C, Wang Y, Li X, Ren L, Zhao J, Hu Y, et al. Clinical features of patients infected with 2019 novel coronavirus in Wuhan, China. Lancet. 2020;395(10223):497-506.

[7] Li Y, Li H, Fan R, Wen B, Zhang J, Cao X, et al. Coronavirus Infections in the Central Nervous System and Respiratory Tract Show Distinct Features in Hospitalized Children. Intervirology. 2016;59(3):163-9.

[8] Oddo M, Carrera E, Claassen J, Mayer SA, Hirsch LJ. Continuous electroencephalography in the medical intensive care unit. Crit Care Med. 2009;37(6):2051-6.

[9] al YLe. Acute Cerebrovascular Disease Following COVID-19: A Single Center, Retrospective, Observational Study. lancet 2020.

[10] Xu Z, Shi L, Wang Y, Zhang J, Huang L, Zhang C, et al. Pathological findings of COVID-19 associated with acute respiratory distress syndrome. The Lancet Respiratory medicine. 2020;8(4):420-2.

[11] Qin C, Zhou L, Hu Z, Zhang S, Yang S, Tao Y, et al. Dysregulation of immune response in patients with COVID-19 in Wuhan, China. Clinical infectious diseases : an official publication of the Infectious Diseases Society of America. 2020.

[12] Cao X. COVID-19: immunopathology and its implications for therapy. Nature reviews Immunology. 2020;20(5):269-70.

[13] Zhao J, Yuan Q, Wang H, Liu W, Liao X, Su Y, et al. Antibody responses to SARS-CoV-2 in patients of novel coronavirus disease 2019. Clinical infectious diseases : an official publication of the Infectious Diseases Society of America. 2020.

[14] Zhang G, Zhang J, Wang B, Zhu X, Wang Q, Qiu S. Analysis of clinical characteristics and laboratory findings of 95 cases of 2019 novel coronavirus pneumonia in Wuhan, China: a retrospective analysis. Respiratory research. 2020;21(1):74.

[15] M Ling MW, et al. Neurological manifestations of Hospitalized Patients with COVID-19 in Wuhan, China: a retrospective case series study. 2020

[16] Ding Y, Wang H, Shen H, Li Z, Geng J, Han H, et al. The clinical pathology of severe acute respiratory syndrome (SARS): a report from China. The Journal of pathology. 2003;200(3):282-9.

[17] AAO-HNS AAoOHaNS. Anosmia, Hyposmia, and Dysgeusia Symptoms of Coronavirus Disease 2020 [cited 2020 23/03/2020]. first [Available from: ; https://www.entnet.org/content/aao-hns-anosmiahyposmia-and-dysgeusia-symptoms-coronavirus-disease.

[18] ENT UK at The Royal College of Surgeons of England 35-43 Lincoln's Inn Fields London WC2A 3PE Tel: 02074048373 | Email: entuk@entuk.org | Web: www.entuk.org1Loss of sense of smell as marker of COVID-19 infection 2020 [cited 2020 March 30]. Available from: I Web: www.entuk.org1Loss of sense of smell as marker of COVID-19 infection.

[19] system Hfh. 2020

[20] Romero-Sanchez CM, Diaz-Maroto I, Fernandez-Diaz E, Sanchez-Larsen A, Layos-Romero A, GarciaGarcia J, et al. Neurologic manifestations in hospitalized patients with COVID-19: The ALBACOVID registry. Neurology. 2020.

[21] Oxley TJ, Mocco J, Majidi S, Kellner CP, Shoirah H, Singh IP, et al. Large-Vessel Stroke as a Presenting Feature of Covid-19 in the Young. N Engl J Med. 2020;382(20):e60. 
[22] A Cervantes DG, T Ford,, J Shulman, A Daneshmand, T Nguyen, K Suchdev, C Takahashi, M Abdennadher, A Saxena, P Anand, K. H. Vincent Lau, A Macone, M Perloff, S Zhu. COVID-19 Neurology Protocols [cited 2020 08/04/2020]. Focused Guidelines ].

[23] Josephson SA, Hills NK, Johnston SC. NIH Stroke Scale reliability in ratings from a large sample of clinicians. Cerebrovascular diseases. 2006;22(5-6):389-95.

[24] Shah VA, Martin CO, Hawkins AM, Holloway WE, Junna S, Akhtar N. Groin complications in endovascular mechanical thrombectomy for acute ischemic stroke: a 10-year single center experience. Journal of neurointerventional surgery. 2016;8(6):568-70.

[25] Powers WJ, Rabinstein AA, Ackerson T, Adeoye OM, Bambakidis NC, Becker K, et al. Guidelines for the Early Management of Patients With Acute Ischemic Stroke: 2019 Update to the 2018 Guidelines for the Early Management of Acute Ischemic Stroke: A Guideline for Healthcare Professionals From the American Heart Association/American Stroke Association. Stroke. 2019;50(12):e344-e418.

[26] Helms J, Kremer S, Merdji H, Clere-Jehl R, Schenck M, Kummerlen C, et al. Neurologic Features in Severe SARS-CoV-2 Infection. N Engl J Med. 2020;382(23):2268-70.

[27] Wada R, Aviv RI, Fox AJ, Sahlas DJ, Gladstone DJ, Tomlinson G, et al. CT angiography "spot sign" predicts hematoma expansion in acute intracerebral hemorrhage. Stroke. 2007;38(4):1257-62

[28] Arabi YM, Harthi A, Hussein J, Bouchama A, Johani S, Hajeer AH, et al. Severe neurologic syndrome associated with Middle East respiratory syndrome corona virus (MERS-CoV). Infection. 2015;43(4):495501.

[29] Chalouhi N, Ali MS, Jabbour PM, Tjoumakaris SI, Gonzalez LF, Rosenwasser RH, et al. Biology of intracranial aneurysms: role of inflammation. J Cereb Blood Flow Metab. 2012;32(9):1659-76.

[30] Seguin A, Galicier L, Boutboul D, Lemiale V, Azoulay E. Pulmonary Involvement in Patients With Hemophagocytic Lymphohistiocytosis. Chest. 2016;149(5):1294-301.

[31] Takeshi M ea. A first Case of Meningitis/Encephalitis associated with SARS-Coronavirus-2. IJID. 2020.

[32] Xu XW, Wu XX, Jiang XG, Xu KJ, Ying LJ, Ma CL, et al. Clinical findings in a group of patients infected with the 2019 novel coronavirus (SARS-Cov-2) outside of Wuhan, China: retrospective case series. BMJ. 2020;368:m606.

[33] Tian S, Hu N, Lou J, Chen K, Kang X, Xiang Z, et al. Characteristics of COVID-19 infection in Beijing. The Journal of infection. 2020;80(4):401-6.

[34] Liu M, He P, Liu HG, Wang XJ, Li FJ, Chen S, et al. [Clinical characteristics of 30 medical workers infected with new coronavirus pneumonia]. Zhonghua jie he he hu xi za zhi = Zhonghua jiehe he huxi zazhi $=$ Chinese journal of tuberculosis and respiratory diseases. 2020;43(3):209-14

[35] WHO. The use of non-steroidal anti-inflammatory drugs (NSAIDs) in patients with COVID-19 2020.

[36] Ling MH, J. Mengdie, W. et al. Neurologic Manifestations of Hospitalized Patients With Coronavirus Disease 2019 in Wuhan, China. JAMA Neurology 2020; Published online April 10, 2020. doi:10.1001/jamaneurol.2020.1127

[37] Zhao H, Shen D, Zhou H, Liu J, Chen S. Guillain-Barre syndrome associated with SARS-CoV-2 infection: causality or coincidence? Lancet Neurol. 2020.

[38] Manji H CA, Brownlee WJ, et al. Neurology in the time of covid-19. J Neurol Neurosurg Psychiatry. 2020.

[39] Visser LH. Critical illness polyneuropathy and myopathy: clinical features, risk factors and prognosis. European journal of neurology. 2006;13(11):1203-12.

[40] Giovannoni G, Hawkes C, Lechner-Scott J, Levy M, Waubant E, Gold J. The COVID-19 pandemic and the use of MS disease-modifying therapies. Multiple sclerosis and related disorders. 2020;39:102073.

[41] Barzegar M, Mirmosayyeb O, Nehzat N, Sarrafi R, Khorvash F, Maghzi AH, et al. COVID-19 infection in a patient with multiple sclerosis treated with fingolimod. Neurology(R) neuroimmunology \& neuroinflammation. 2020;7(4). 